\title{
A SMART BASE RESTRAINT FOR WIND TURBINES TO MITIGATE UNDESIRED EFFECTS DUE TO STRUCTURAL VIBRATIONS
}

\author{
N. Caterino ${ }^{1}$, C.T. Georgakis ${ }^{2}$, M. Spizzuoco ${ }^{3}$ and A. Occhiuzzi ${ }^{4}$ \\ ${ }^{1}$ Department of Civil Engineering, University of Naples "Parthenope" \\ Centro Direzionale di Napoli, Isola C4, 80143, Naples, Italy \\ nicola.caterino@uniparthenope.it \\ ${ }^{2}$ Department of Civil Engineering, Technical University of Denmark (DTU) \\ Building 118, 2800 Kgs. Lyngby, Denmark \\ cg@byg.dtu.dk \\ ${ }^{3}$ Department of Structures for Engineering and Architecture, University of Naples Federico II \\ Via Claudio 21, 80125, Naples, Italy \\ spizzuoc@unina.it \\ ${ }^{4}$ Construction Technologies Institute, National Research Council (CNR) \\ Viale Lombardia, 49, 20098, San Giuliano Milanese (MI), Italy \\ occhiuzzi@itc.cnr.it
}

Keywords: Semi-Active Control, Wind Turbine, Magnetorheological Damper.

\begin{abstract}
Concerns in the last decades of the negative impact of the use of fossil fuels on the environment has lead to a boom in the production of wind turbines. To take advantage of the smoother stronger winds at height, wind turbine heights are progressively increasing. This has led to an increased demand to control tower forces. The application of a semi-active (SA) control system is herein proposed and discussed. Its aim is to limit bending moment demand at the base of a wind turbine by relaxing the base restraint of the turbine's tower, without increasing the top displacement. This is done thanks to the sharp increase of the dissipated energy in selected intervals of time and an adaptive change in tower dynamic properties. This SA control system reproduces a variable restraint at the base that changes in real time its mechanical properties according to the instantaneous response of the turbine's tower. This smart restraint is made of a central smooth hinge, elastic springs and SA magnetorheological dampers driven by a control algorithm properly designed for the specific application. A commercial $105 m$ tall wind turbine has been assumed as a case study. Several numerical simulations have been performed with reference to an extreme load, aimed at establishing a procedure for the optimal calibration of the control algorithm according to the specific case, finally proving the actual potential of the proposed control technique in reducing the structural demand with respect to the "fixed base" structure.
\end{abstract}




\section{INTRODUCTION}

Wind turbine manufacturers have become ever more interested in methods for reducing or limiting tower base moments for turbines of increasing height. There are two main reasons for this. First, the tower diameters at the base are increasing beyond the point where they can be fabricated off-site and shipped to the location of installation. This significantly increases construction costs and complexity. Second, the tower diameters and associated base moments of newer wind turbines far exceed those of existing wind turbines that the newer ones hope to replace. Therefore, existing foundations cannot be utilized and new or strengthened foundations need to be constructed.

The maximum base moments of a wind-turbine are generated by few very specific, and in some cases improbable, load cases. These include the load cases which involve extreme gusts of wind combined with emergency shut-down procedures and extreme wind loading in a parked configuration. All of the extreme load cases generate maximum expected tower base moments via short duration impulse loading - something which cannot be combated using traditional damping devices. Damping is generally considered ineffective for impact type loading. Instead, a variable tower stiffness and damping approach needs to be followed. Therefore, in this paper, the concept of an adaptive wind turbine tower is examined, where the adaptation is realized through the implementation of a semi-active (SA) control system.

Chen and Georgakis [1] performed an experimental analysis of a 1/20-scale wind tower model equipped with a passive rolling-ball damper to reduce vibrations. Such damper consists of a glass container placed at the top of the model and having one or more steel balls inside. Different configurations have been tested, changing the geometry of the container (one or two layers) and the number of balls (one to six) placed inside, showing a significant reduction of the peak value and standard deviation of top displacement and base bending moment. The same authors [2] tested the same model using water rather than steel balls inside the glass container, i.e. realizing a spherical tuned liquid damper. The optimal degree of filling with water (1-2\% of the total generalized mass of the system) has been found as leading to the maximum reduction of structural demand.

About active/semi-active control strategies from literature related to wind turbines, Karimi et al. [3] and Luo et al. [4] propose a SA control technique for floating wind turbines with TLCD. This device, generally used as a passive damper, turns into a SA device using a controllable valve. The orifice opening is real time adapted according to the structure response and loading conditions, with a control logic based on a $\mathrm{H}_{\infty}$ feedback methodology. Lackner and Rotea [5] investigate the effectiveness of an optimal passive TMD and of a hybrid mass damper (HMD, i.e. a TMD improved with the addition of a controlled force actuator) in reducing fatigue loads due to bending moment at the base of the tower, showing a percentage reduction of about $10 \%$ and $30 \%$ respectively due to each of the two proposed systems. Kirkegaard et al. [6] have been the first to explore the use of magnetorheological (MR) dampers to control a wind turbine, assuming such type of smart device to be installed, in a vertical position, between the base and the top of the tower. Even hard to be implemented in a real case, the numerical simulations show good results. Experimental results are also made available by the authors, unfortunately referred to the passive use (constant voltage fed to the MR damper) of the device only.

The authors recently proposed a SA control system based on the application of MR devices to realize a time-variant base restraint whose 'stiffness' can be driven in real time by a properly written control logic [7]. The high promptness of response of the SA control chain based on the use of MR dampers and of an appropriate electronic and power equipment has been experimentally demonstrated [8], showing that unavoidable delays however are always less than 10 
ms, thus negligible compared to the long period of the flexible structures under examination. This is one of the aspects strongly encourage the use of such technology for the application in question. The controller has to be programmed to instantaneously calibrate the MR devices installed at the base of the tower in order to reduce the base bending moment, relaxing in selected intervals of time the base restraint. Again, the control logic has to hold the top displacement within acceptable values so as to avoid significant, detrimental second order effects. At the laboratory of the Denmark Technical University (DTU) in Copenaghen, some shaking table tests of a wind turbine tower model semi-actively controlled as above has been recently performed by the authors. After the formulation of the above idea, a finite element model of the structure has been calibrated so as to develop several numerical simulations addressed to optimally calibrate the control logic properly designed for such kind of applications.

\section{A VARIABLE BASE RESTRAINT FOR WIND TURBINE TOWERS: CONTROL ALGORITHM}

A smart restraint at the base of high-rise wind turbine towers is proposed with the aim of reducing wind induced structural demand by exploiting magnetorheological devices. This is schematically shown in Figure 1, where the uncontrolled wind turbine, fully restrained at the base, is modeled as a single degree of freedom dynamic system (Figure 1(a)), having top mass $m$, stiffness $k_{T}$ and inherent damping $c_{T}$. In order to control the structural demand, the authors proposed to replace the perfectly rigid base restraint with a controllable one that is able to instantaneously become more or less "stiff", during the motion. Figure 1(b) just sketches the materialization of this idea by a smooth hinge, with a rotational spring (of stiffness $k_{\phi}$ ) and a rotational variable damper whose damping constant $c_{\phi}$ can be driven in real time by a control algorithm. The same result can be obtained in practice by mounting two vertical linear springs $\left(k_{s}\right)$ placed at a certain distance $\left(l_{s}\right)$ from the hinge and two vertical SA dampers $\left(c_{d}\right)$ at a distance $l_{d}$ from the central hinge (Figure 1(c)).

SA MR dampers were considered as smart devices within the proposed control system: when a low value is imposed to the base damping, the base restraint is less 'stiff', so that the structure's restraint is able to relax by converting its potential energy into kinetic energy, and the bending moment at the base is reduced. A direct consequence of controlling the demand of base bending stress could be an increase of top displacement demand; therefore, the SA base control system was thought to reduce base stress, by restraining the increase of top displacements within certain limits to control second order effects. A specific bang-bang control algorithm has been formulated by the authors [7] to instantaneously decide the system's base configuration: it switches back and forth from an "OFF" state (intensity of current $i=i_{\min }$, i.e. the minimum current set to be given to the dampers) to an "ON" state ( $i=i_{\max }$, i.e. the maximum assumed value for the current) according to a logic aiming to control both the base stress and the top displacement. Therefore, the control algorithm was so formulated:
1) if $|\sigma(t)|<\sigma_{\text {lim }}$ $\rightarrow i(t)=i_{\max }$
2) if $|\sigma(t)| \geq \sigma_{\lim }$ and $|x(t)|<x_{\lim }$$$
\rightarrow i(t)=0
$$
3) if $|\sigma(t)| \geq \sigma_{\lim }$ and $|x(t)| \geq x_{\lim }$
4) if $|\sigma(t)| \geq \sigma_{\lim }$ and $|x(t)| \geq x_{\lim }$
and $x(t) \cdot \dot{x}(t)>0$
$\rightarrow i(t)=i_{\max }$
and $x(t) \cdot \dot{x}(t) \leq 0 \quad \rightarrow i(t)=0$

where $\sigma(t), x(t)$ and $(t)$ are respectively the value of stress at the base, top displacement and top velocity at the instant of time $t$. In other words, the controller keeps 'stiffer' the base restraint until the stress exceeds the limit value $\sigma_{\text {lim }}$ (expression 1) of Eq. (1)), whereas 'relaxes' it ("OFF" state of the dampers) when this limit is overpassed and the displacement falls within 
the limit of acceptability $x_{\lim }$ (expression 2) of Eq. (1)). When both stress and displacement are beyond the respective threshold values, the controller switches "ON" the dampers if the displacement is going towards a larger value (so trying to damp or invert the displacement's trend; expression 3) of Eq. (1)), otherwise it switches "OFF" the MR devices to make them collaborating to both stress and displacement reduction. Figure 2 schematically describes the above defined logic: the decision of the controller (switch "ON" or switch "OFF") depends on the occurrence of each of the four possible combinations regarding the value of base stress and top displacement. The application of the proposed control algorithm requires the definition of rational criteria to optimally calibrate the parameters involved in $\left(i_{\min }, i_{\max }, \sigma_{\text {lim }}\right.$ and $\left.x_{\text {lim }}\right)$. A large numerical campaign has been performed with reference to a case study structure, aiming to investigate the role each parameter has regarding the structural response, and to learn a possible procedure to calibrate them aiming at achieving the maximum reduction of stresses and displacements.

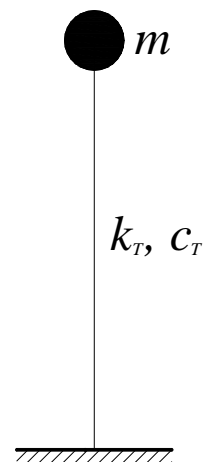

(a)

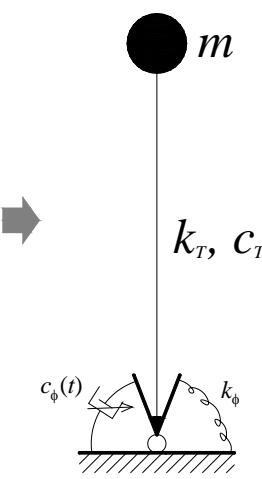

(b)

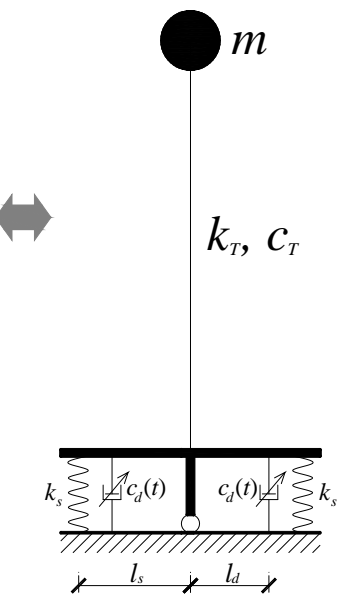

(c)

Figure 1: Basic idea of SA control of a wind turbine via MR dampers.

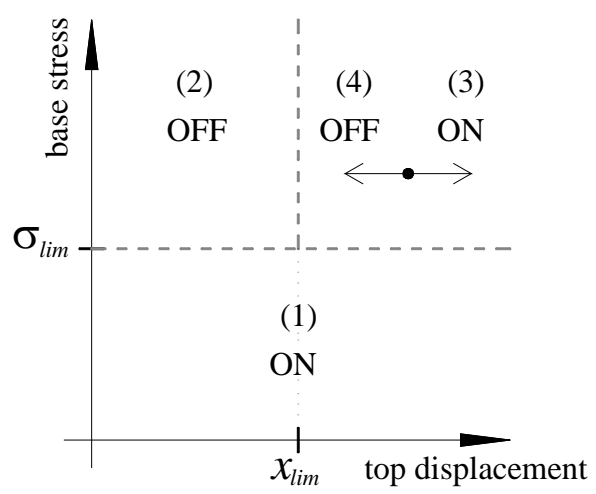

Figure 2: The logic behind the controller (symbols refer to Eq. (1)).

\section{CALIBRATION OF THE SA CONTROLLER: A CASE STUDY}

A possible calibration procedure is herein proposed to provide the optimal choice of values to be assigned to the parameters involved in the control algorithm. The first step is generating a finite element model of the structure to be examined, able to reproduce both fixed base (FB) and SA controlled configurations. With reference to a given wind load, the structural response in the FB case has to be determined. Then a wide number of SA numerical simulations has to 
be designed and performed. Analyzing the results using a constrained optimization approach allows to single out the optimal configuration of the controller able to achieve the maximum reduction of base stress while not causing increasing of top displacement in respect to the FB case. This procedure is practically applied in the following with reference to a specific case study.

\subsection{Case Study}

The case study structure is a $1 / 20$-scale structural model of a prototype real wind turbine. The reference real structure is a $3 \mathrm{MW}$ wind turbine with horizontal power transmission axle, $102.4 \mathrm{~m}$ tall, with a variable hollow circular cross section whose external diameter is variable from $2.30 \mathrm{~m}$ (top) to $4.15 \mathrm{~m}$ (bottom). Chen and Georgakis [1] demonstrated its dynamic equivalence (in terms of equivalent flexural stiffness) with a single degree of freedom (SDOF) structural system made up of a tapered tubular cantilever beam with a concentrated mass at the top. The scaled mock-up structure is characterized by a $5.12 \mathrm{~m}$ high vertical tube with uniform cross section $\Phi 133 / 4$ (133 $\mathrm{mm}$ is the external diameter, $4 \mathrm{~mm}$ the thickness), and a lumped mass of $280 \mathrm{~kg}$ placed at the top (Figure 3).

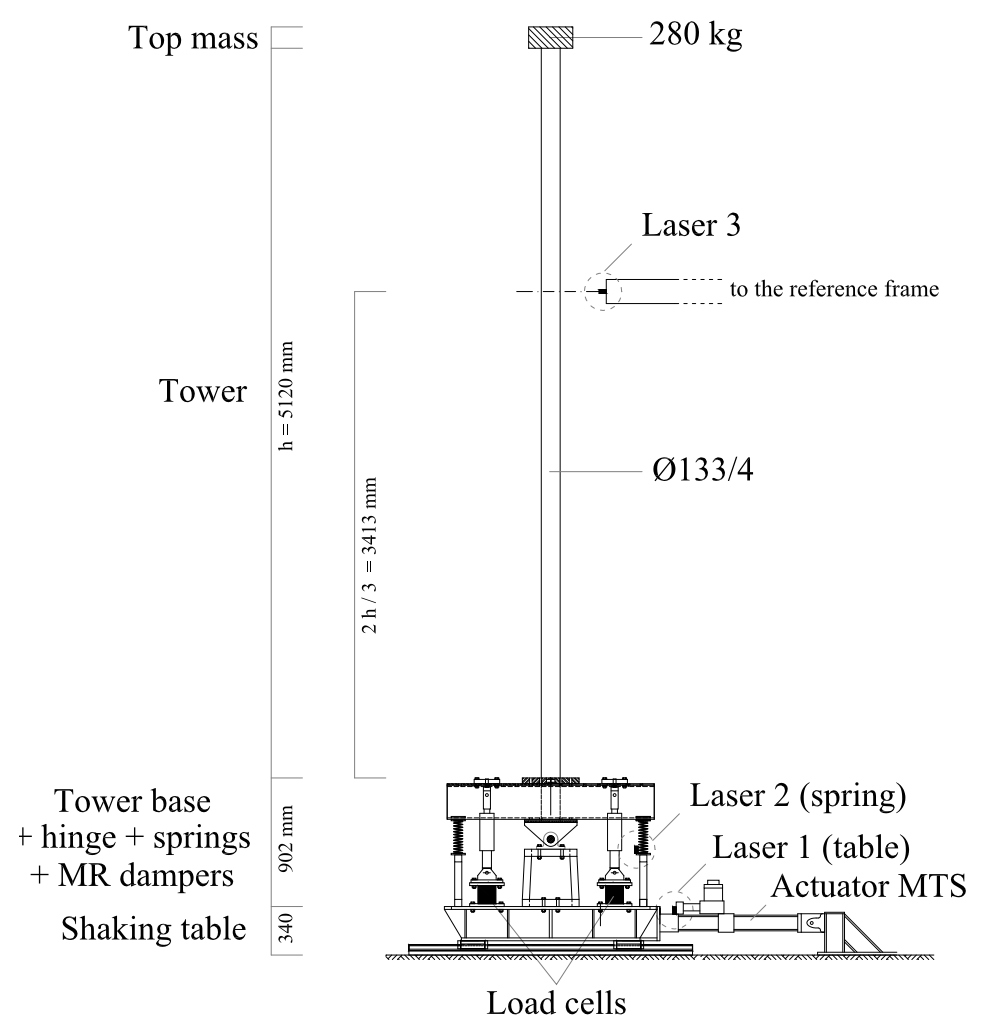

Figure 3: Case study model and experimental setup at the DTU laboratory.

The base of the model is highly stiff and is supported in the middle by a cylindrical steel hinge. On both sides of the base, one cylindrical spring ( $89 \mathrm{kN} / \mathrm{m}$ stiff) and one MR damper are installed. The assembly "elastic springs + SA MR dampers", placed in parallel at the base of the tower, just represents the smart base restraint herein proposed to control the dynamic behavior of the structure. An extreme operating gust loading has been considered in the following as reference wind action: a sharp increase, then decrease in wind speed within a short period of time. Chen and Georgakis [1] defined an equivalent base acceleration time history 
(Figure 4), that is the base input that would provide the same top mass response of the real fixed base structure subjected to the wind action.

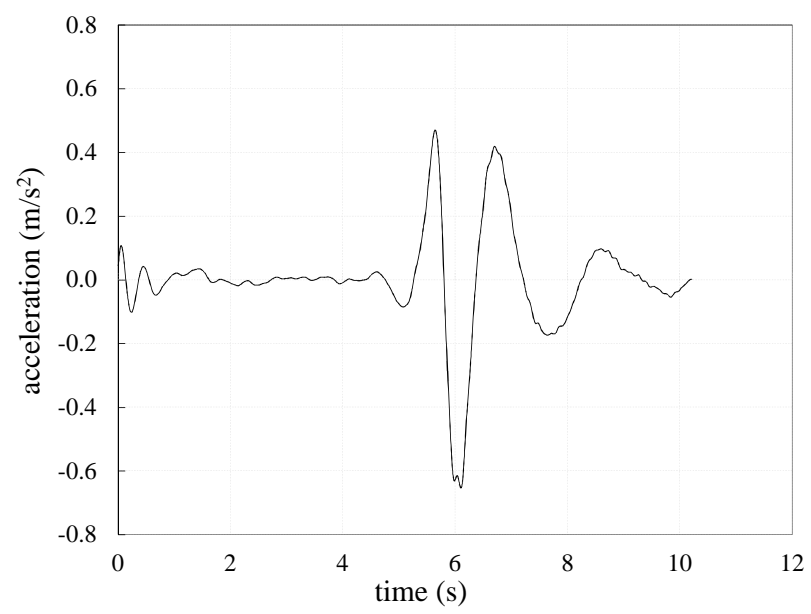

Figure 4: Equivalent base acceleration time history for the wind load case.

\subsection{Numerical model}

A finite element model has been generated in Matlab environment to simulate the dynamic behavior of the case study structure. It consists in 37 elements: 36 elements simulate the tower with uniform diameter $(133 \mathrm{~mm})$ and thickness $(4 \mathrm{~mm})$ along the height, while the last element $\left(37^{\text {th }}\right)$ is more rigid and represents the connection of the top of the tower to the barycenter of the nacelle. The rotor and the aerodynamics have not been included in the model due to its complexity, and the nacelle and its internal components are represented by a concentrated mass at the top of the structure, with no dynamic interaction considered. Such mass is added in the global mass matrix at the translational degree of freedom at the top of the tower.

The base support has been modeled as in Figure 5, that is by a rotational spring $k_{\text {spring }}$ and a Maxwell element (representing the MR dampers) working in parallel. The value for $k_{s p r i n g}$ $(7.5 \mathrm{e} 4 \mathrm{Nm} / \mathrm{rad}$ ) has been easily derived known the stiffness of the two linear springs and their distance from the center of rotation (hinge).

The Maxwell element, as known, consists of a spring $k_{\text {Maxwell }}$ and a linear viscous damper $c_{\text {Maxwell }}$ in series. The controllable part of this device is represented by the constant $c_{\text {Maxwell, }}$ while $k_{\text {Maxwell }}$ has been simply assumed high enough $(1.5 \mathrm{e} 6 \mathrm{Nm} / \mathrm{rad})$ so as to behave like a rigid link. Two different values of $c_{\text {Maxwell }}\left(c_{o n}, c_{\text {off }}\right)$ have been determined so as to reproduce the dissipative capability of MR dampers respectively in the "ON" and "OFF" states. These two opposite configurations of the MR dampers are assumed to be those of the experimental campaign cited above, respectively corresponding to $i=i_{\min }=0 \mathrm{~A}$ and $i=i_{\max }=1 \mathrm{~A}$. The MR dampers considered to calibrate the Maxwell device properties are those adopted for the test at DTU also [9]. Suitable numerical models for such devices are described in [10]. The values of $c_{o n}$, $c_{\text {off }}$ have been experimentally calibrated and resulted to be $c_{\text {on }}=1 \mathrm{e} 7 \mathrm{Nms} / \mathrm{rad}$ and $c_{\text {off }}=2 \mathrm{e} 3$ $\mathrm{Nms} / \mathrm{rad}$. 


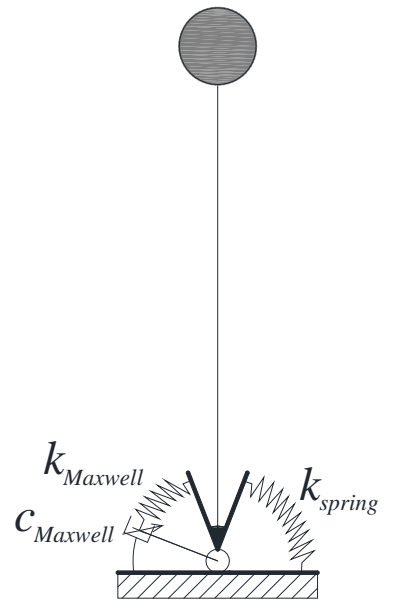

Figure 5: Representation of the base restraint within the FE model of the SA controlled structure.

\subsection{Numerical simulations}

A number of 100 numerical tests have been performed with reference to the above FEM model in SA configuration. This is the total number of different combinations of stress $\left(\sigma_{\text {lim }}\right)$ and displacement $\left(x_{\text {lim }}\right)$ limits that have been tested, chosen within the ranges $[5,50] \mathrm{MPa}$ and $[5,50] \mathrm{mm}$ with a step of $5 \mathrm{MPa}$ and $5 \mathrm{~mm}$, respectively. Performance indices have been assumed to quantitatively summarize the structural response in the examined cases.

These allow to compare the effectiveness of the SA control strategy for each of the above settings for the controller, then to select the optimal calibration of the latter. They are:

- ratio of maximum bending stress $\sigma_{\max }$ to limit value $\sigma_{\text {lim }}$ assumed to calibrate the controller $\left(\sigma_{\max } / \sigma_{\text {lim }}\right)$;

- ratio of maximum bending stress in SA to fixed base (FB) conditions ( $\left.\sigma_{\max } / \sigma_{\max , \mathrm{FB}}\right)$;

- ratio of maximum top displacement in SA to FB conditions $\left(x_{\max } / x_{\max , \mathrm{FB}}\right)$.

Moreover, to better interpret the results, the following two additional information have been gathered from each simulation performed:

- total amount of time in which the MR damper has been switched off by the controller ( $\left.\mathrm{t}_{\mathrm{off}}\right)$;

- total number of switches (on $\rightarrow$ off and vice versa) commanded to the variable device $\left(\mathrm{n}_{\mathrm{sw}}\right)$.

The ratio $\sigma_{\max } / \sigma_{\text {lim }}$ is assumed so as to check if and how the controller has been able to limit the bending stress to the desired value $\sigma_{\text {lim }}$, while the ratio $x_{\max } / x_{\text {lim }}$ has not been assumed as parameter for comparison because it is not significant to the same extent given that $x_{\text {lim }}$ has a reduced impact on the controller operation. The indices $\sigma_{\max } / \sigma_{\max , \mathrm{FB}}$ and $x_{\max } /$ $x_{\mathrm{max}, \mathrm{FB}}$ express the effectiveness of the controller in reducing the structural response with respect to the FB conditions. Values less than one are desired, in fact they reflect the main purpose of the control strategy. The indices $t_{\text {off }}$ and $n_{s w}$ give a quantitative idea about the activity of the MR damper during each test. When the smart device is set to "ON", it is not very far from acting as a rigid link. Therefore, the above $t_{\text {off }}$ gives also a measure of the overall duration of the dissipation phase.

\section{DISCUSSION ABOUT RESULTS AND CONCLUSIONS}

The constrained optimization of the controller is performed according to the condition:

$$
\min \left(\sigma_{\max } / \sigma_{\max , F B}\right) \text { subject to } x_{\max } / x_{\max , F B} \leq 1
$$


that is, aiming to achieve the greatest reduction of the base stress (objective function) and, at the same time, a top displacement (constraint function) no higher than that in uncontrolled FB conditions.

Analyzing diagrams (a) in Figure 6, it emerges that the SA control of the case study wind turbine is always beneficial in terms of reduction of base stress with respect to the fixed base scheme. Considered the whole set of examined cases, the maximum base stress reduction results to be around $80 \%$, and corresponds to the case $\left(\sigma_{\text {lim }}, x_{\text {lim }}\right)=(5 \mathrm{MPa}, 25 \mathrm{~mm})$. The maximum top displacement reduction is about $35 \%$, for $\left(\sigma_{\text {lim }}, x_{\text {lim }}\right)=(10 \mathrm{MPa}, 15 \mathrm{~mm})$. The worst case, i.e. that corresponding to the maximum amplification of $x(+40 \%)$ with respect to the FB case is $\left(\sigma_{\text {lim }}, x_{l i m}\right)=(35 \mathrm{MPa}, 50 \mathrm{~mm})$.

Higher operation of the SA controller for smaller value of $\sigma_{\text {lim }}$, as expected, can be deduced from Figure 6(d). Figure 6(c) instead highlights that response values in terms of maximum base stress $\sigma_{\max }$ are practically always included in the interval $\left[\sigma_{l i m}, 2 \sigma_{l i m}\right]$, with the exception of those cases where both very small values are fixed both for $\sigma_{\text {lim }}$ and $x_{\text {lim }}$.

Actually, about half (53\%) of the examined configurations of the controller leaded to undesired response in top displacement, i.e. values of $x_{\max }$ larger than $x_{\max , F B}$. Figure 7 helps to understand better which are the combinations of limit values $\sigma_{\text {lim }}$ and $x_{\text {lim }}$ that give undesired response in displacement. It results that, when values of $\sigma_{\text {lim }}$ greater than $30 \mathrm{MPa}$ are adopted (i.e. roughly greater than half of the $\sigma_{\max , \mathrm{FB}}$ value), there is no chance to reduce displacements in respect to the FB case. The reason is related to the fact that in such cases the SA operations are really limited, as from Figure 6(d) clearly emerges. Therefore, the dissipation phases are concentrated in much small intervals of time, not effective in reducing significantly the response in displacement. Vice versa, when smaller values of $\sigma_{l i m}$ are used (i.e. less than 30 $\mathrm{MPa}$ ), the reduction or amplification of $x_{\max }$ in respect to $x_{\max , \mathrm{FB}}$ also depends on the assumed value for $x_{\text {lim. }}$. Also in this case, it seems that selecting values for $x_{\text {lim }}$ less than $0.5 x_{\text {max,FB }}$ leads always to good results in terms of displacement response.

According to the criterion defined in the condition above, the optimal configuration of the control algorithm corresponds to the case $\left(\sigma_{\text {lim }}, x_{\text {lim }}\right)=(5 \mathrm{MPa}, 25 \mathrm{~mm})$ since it leads to the maximum response reduction (about 80\%) in base stress, and also to a reduction (about 30\%) of displacement in respect to the FB case.

Therefore, preliminary conclusions about a possible way to optimally calibrate the controller could be drawn suggesting to assume, for $\sigma_{\lim }$ and $x_{\text {lim }}$, values respectively around $0.1 \sigma_{\max , \mathrm{FB}}$ and $0.5 x_{\max , \mathrm{FB}}$. Moderately low values of $\sigma_{\lim }\left(25-50 \%\right.$ of $\left.\sigma_{\max , \mathrm{FB}}\right)$ leaded to increased top displacements, even higher than the reference value $x_{\max , \mathrm{FB}}$. A trend reversal for values of $\sigma_{\text {lim }}$ even more smaller (10-20\% of $\left.\sigma_{\max , \mathrm{FB}}\right)$ has been registered, leading them to significant reduction of both base stress and top displacement, as said above, due to the higher operation of the controller and, consequently, to the sharp increase of dissipated energy due to the larger rocking of the base.

The above results cannot be directly generalized, since clearly dependent on the specific wind load case and turbine model assumed for the analyses. On the contrary, the conceptual findings they allowed to gather are always valid and will be exploited by the authors to program further investigations aimed at consolidating those outcomes and to finally determine the practical implications they could have. Future further research about this topic will have to consider a larger set of wind load cases, different for magnitude, duration and frequency content, to understand if and how the optimal calibration of the control algorithm depends on the characteristics of the external action. 

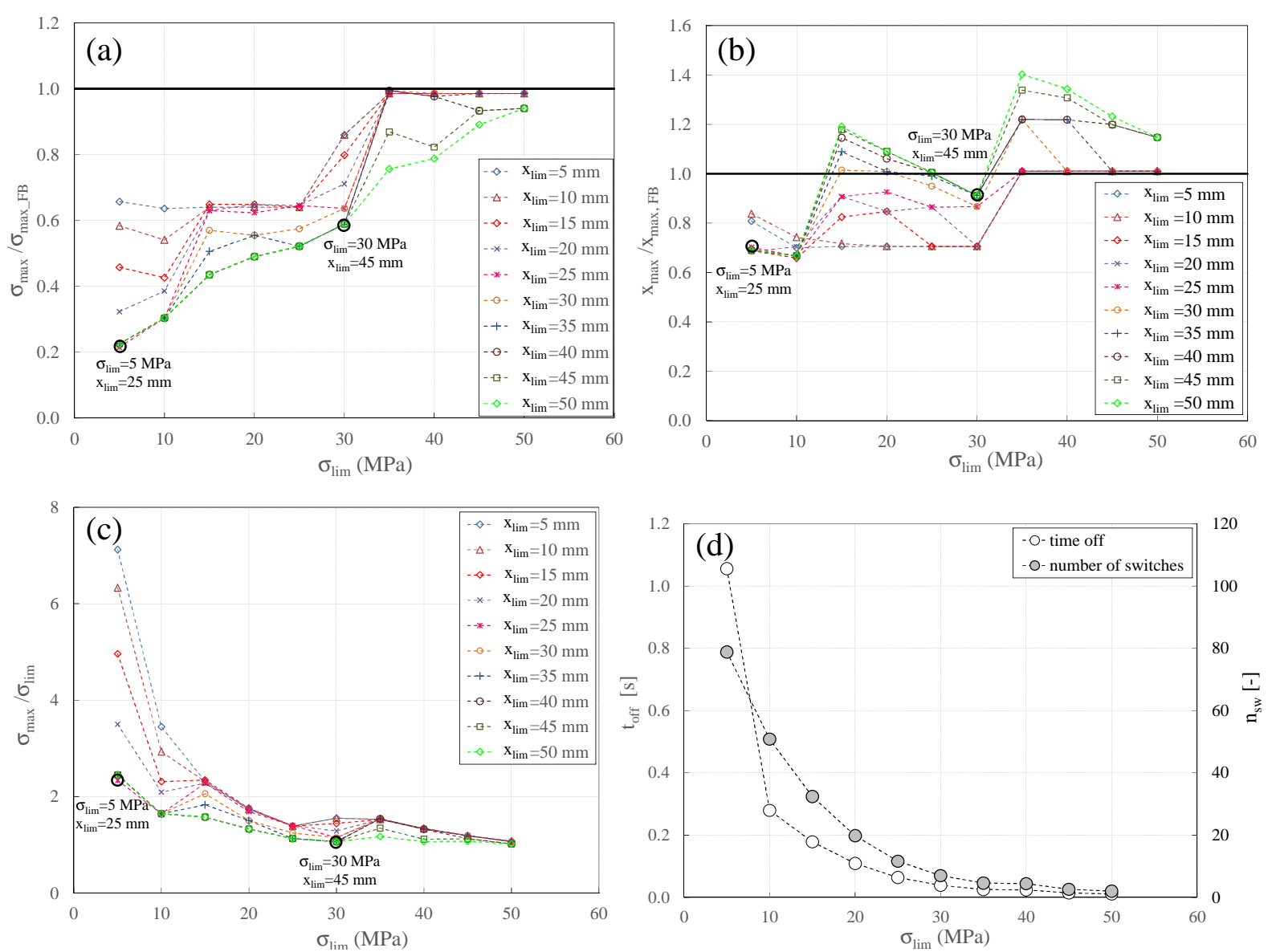

Figure 6: Performance indices for the 100 configurations of the SA controller.

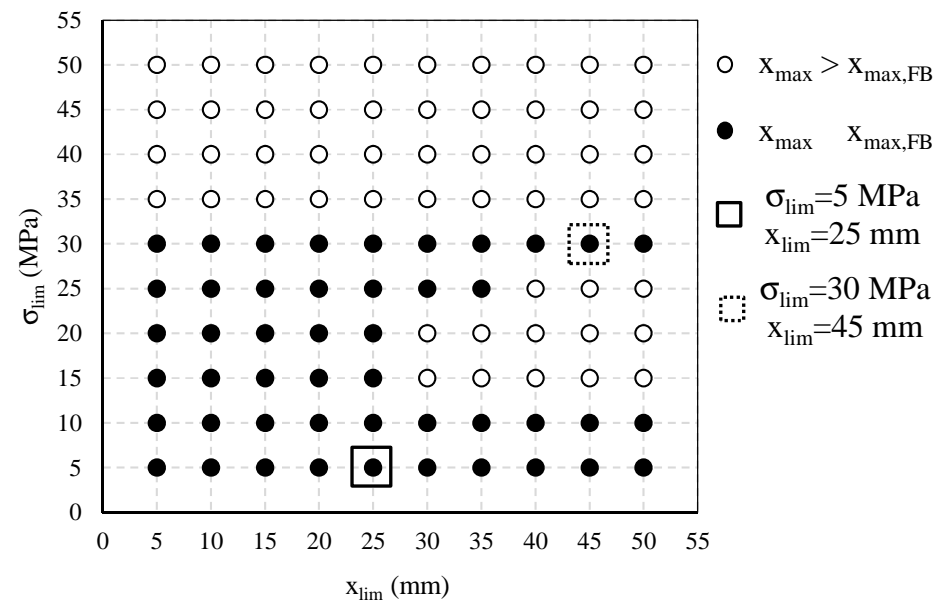

Figure 7: Configurations of the controller corresponding to acceptable (black filled circle) and excessive (empty circle) top displacement response.

\section{ACKNOWLEDGEMENTS}

The presented activity has been partially funded by the Italian consortium Reluis in the framework of the 2015 and 2016 research projects supported by the Italian Department for Civil Protection. This support is gratefully acknowledged. 


\section{REFERENCES}

[1] J. Chen, C.T. Georgakis, Tuned rolling-ball dampers for vibration control in wind turbines. Journal of Sound and Vibration, 332, 5271-5282, 2013, dx.doi.org/10.1016/j.jsv.2013.05.019.

[2] J. Chen, C.T. Georgakis, Spherical tuned liquid damper for vibration control in wind turbines. Journal of Vibration and Control, 21(10), 1875-1885, 2015, doi: $10.1177 / 1077546313495911$.

[3] H.R. Karimi, M. Zapateiro, N. Luo, Semiactive vibration control of offshore wind turbine towers with tuned liquid column dampers using $\mathrm{H} \infty$ output feedback control. Proceedings of the IEEE International Conference on Control Applications, Yokohama, Japan, 2010.

[4] N. Luo, C.L. Bottasso, H.R. Karimi, M. Zapateiro, Semiactive Control for floating offshore wind turbines subject to aero-hydro dynamic loads. Proceedings of the International Conference on Renewable Energies and Power Quality, Las Palmas de Gran Canaria, Spain, 2011.

[5] M. Lackner, M. Rotea, Structural control of floating wind turbines. Mechatronics, 21, 704-719, 2011, doi:10.1016/j.mechatronics.2010.11.007.

[6] P.H. Kirkegaard, S.R.K. Nielsen, B.L. Poulsen, J. Andersen, L.H. Pedersen, B.J. Pedersen, Semi-active vibration control of a wind turbine tower using an MR damper. $\mathrm{H}$. Grundmann, G.I. Schuëller, Rotterdam: Balkema Publishers, A.A. / Taylor \& Francis The Netherlands, 1575-1580, 2002.

[7] N. Caterino, C.T. Georgakis, F. Trinchillo, A. Occhiuzzi, A semi-active control system for wind turbines. In: N. Luo, Y. Vidal, L. Acho, Wind Turbine Control and Monitoring, Springer International Publishing, 375-407, Advances in Industrial Control, 2014, doi: 10.1007/978-3-319-08413-8_13.

[8] N. Caterino, M. Spizzuoco, A. Occhiuzzi, Promptness and dissipative capacity of MR dampers: experimental investigations, Structural Control and Health Monitoring, 2013, 20(12), 1424-1440, doi: 10.1002/stc.1578.

[9] N. Caterino, Semi-active control of a wind turbine via magnetorheological dampers. Journal of Sound and Vibration, 345, 1-17, 2015, doi:10.1016/j.jsv.2015.01.022.

[10] N. Caterino, M. Spizzuoco, A. Occhiuzzi, Understanding and modelling the physical behaviour of magnetorheological dampers for seismic structural control. Smart Material and Structures, 20(6), 2011, doi:10.1088/0964-1726/20/6/065013. 